\title{
Laser polarization fluorescence of the networks of optically anisotropic biological crystals
}

\author{
Y.A. Ushenko ${ }^{* a}$, A.V. Dubolazov ${ }^{\mathrm{a}}$, A.P. Angelsky ${ }^{\mathrm{a}}$, M.I. Sidor ${ }^{\mathrm{a}}$, \\ G.B. Bodnar ${ }^{\text {b }, ~ G . ~ K o v a l ~}{ }^{\text {b }}$, N.I. Zabolotna ${ }^{\mathrm{c}}$, A. Smolarz ${ }^{\mathrm{d}}$, M. Sh. Junisbekov ${ }^{\mathrm{e}}$ \\ ${ }^{a}$ Correlation Optics Department, Chernivtsi National University, 2 Kotsyubinsky Str., Chernivtsi \\ 58012, Ukraine; ${ }^{b}$ Department of Pediatrics and Medical Genetics, Bukovinian State Medical \\ University, 3 Theater Sq., Chernivtsi 58000, Ukraine; ${ }^{c}$ Department of Laser and Optoelectronics \\ Technique, Vinnitsa National Technique University, 93 Khmelnitsky Road, Vinnitsa 21021, \\ Ukraine; ${ }^{\mathrm{d}}$ Lublin University of Technology, Nadbystrzycka 38A, 20-618 Lublin, Poland; \\ ${ }^{\mathrm{e}}$ Taraz State University named after M.Kh.Dulaty, Tole bi Street 60, Taraz, Kazakhstan
}

\begin{abstract}
The present work is devoted to investigation of mechanisms of optical anisotropy of biological tissues polycrystalline networks and laser polarization fluorescence. The model of complex optical anisotropy, which takes into account both linear and circular birefringence, as well as linear and circular dichroism of fibrillar networks of histological sections of women reproductive sphere is proposed. The data of statistical, correlation and fractal processing of coordinate distributions of laser polarization fluorescence is provided. The technique of azimuthally invariant Mueller-matrix mapping of laser polarization fluorescence of protein networks in the tasks of differentiation of benign and malignant tumors of uterus wall is elaborated.
\end{abstract}

Keywords: polarization, polarimetry, Mueller matrix, fluorescence, biological tissue

\section{INTRODUCTION}

Biological tissues represent structurally inhomogeneous optically anisotropic media with absorption. To describe the interaction of polarized light with such sophisticated systems, more general approximation based on Mueller-matrix formalism are required. Nowadays many practical techniques based on measuring and analyzing the Mueller-matrices of the samples under investigation are being used in biological and medical researches ${ }^{1-10}$. During recent $10-15$ years a separate direction - laser polarimetry - has been formed in matrix optics ${ }^{11-20}$. On its basis the interconnections between the set of statistical moments of the $1^{\text {st }}-4^{\text {th }} \operatorname{order}^{11,12}$, correlation ${ }^{15-17}$, fractal ${ }^{18,19}$ and singular ${ }^{20}$ parameters were determined, which characterize the distributions of Mueller matrix elements and the parameters of linear birefringence of fibrillar protein networks of human biological tissues. The diagnostics of pathological changes of skin derma, epithelial and connective tissues of the women's reproductive organs, etc. was realized on this basis ${ }^{12,14,15,18,20}$.

Simultaneously with the polarimetry techniques the methods based on the diagnostic application of the effects fluorescence of protein molecules and their complexes are being intensely developed ${ }^{21}$.

At the same time there practically no data concerning polarization manifestations of fluorescence effects in biological tissues in modern literature. Therefore, the task of complex uniting the diagnostic potentialities of the techniques of laser polarimetry and laser fluorescence proves to be topical.

In this research the model of complex optical anisotropy, possessed by protein networks of the tissues of women's reproductive sphere organs is suggested, and on this ground the method of Mueller-matrix mapping of laser polarization fluorescence of histological sections of biopsy of benign (polypus) and malignant (adenocarcinoma) tumors of uterus wall is developed.

*yuriyu@gmail.com

Optical Fibers and Their Applications 2012, edited by Ryszard S. Romaniuk, Waldemar Wójcik, Proc. of SPIE Vol. 8698, 869809 · (C) 2012 SPIE · CCC code: 0277-786/12/\$18 · doi: 10.1117/12.2019350 


\section{CONCISE THEORY}

The description of laser polarization fluorescence of biological tissues is based on the following model idea of optical anisotropy of protein networks: fluorescence of protein network is grounded on the mechanisms of optically anisotropic absorption (linear and circular dichroism) and the mechanisms of phase anisotropy (linear and circular birefringence) which modulate the secondary radiation of biological molecules and their structures.

\subsection{The mechanisms of optically anisotropic absorption}

Amino acids and polypeptide circuits (initial structure of the protein) formed by them, that possess linear dichroism. Optical manifestations of such a mechanism are quite completely described by the following Mueller matrices:

$$
\{\Psi\}=\left\|\begin{array}{cccc}
1 & \varphi_{12} & \varphi_{13} & 0 \\
\varphi_{21} & \varphi_{22} & \varphi_{23} & 0 \\
\varphi_{31} & \varphi_{32} & \varphi_{33} & 0 \\
0 & 0 & 0 & \varphi_{44}
\end{array}\right\|,
$$

where

$$
\varphi_{i k}=\left\{\begin{array}{l}
\varphi_{12}=\varphi_{21}=(1-\Delta \tau) \cos 2 \rho \\
\varphi_{13}=\varphi_{31}=(1-\Delta \tau) \sin 2 \rho \\
\varphi_{22}=(1+\Delta \tau) \cos ^{2} 2 \rho+2 \sqrt{\Delta \tau} \sin ^{2} 2 \rho \\
\varphi_{23}=\varphi_{32}=(1-\Delta \tau) \sin 2 \rho ; \\
\varphi_{33}=(1+\Delta \tau) \sin ^{2} 2 \rho+2 \sqrt{\Delta \tau} \cos ^{2} 2 \rho ; \\
\varphi_{44}=2 \sqrt{\Delta \tau} .
\end{array}\right.
$$

Here $\Delta \tau=\frac{\tau_{x}}{\tau_{y}},\left\{\begin{array}{l}\tau_{x}=\tau \cos \rho ; \\ \tau_{y}=\tau \sin \rho\end{array}, \tau_{x}, \quad \tau_{y}\right.$ - absorption coefficients of linearly polarized orthogonal components of the light beam amplitude.

Availability of complex spiral-like polypeptide protein structures or their combinations (tertiary structure) forms circular dichroism. Optical manifestations of such configuration peculiarities are characterized by Mueller matrix

$$
\{\Phi\}=\left\|\begin{array}{cccc}
1 & 0 & 0 & \phi_{14} \\
0 & \phi_{22} & 0 & 0 \\
0 & 0 & \phi_{33} & 0 \\
\phi_{41} & 0 & 0 & 1
\end{array}\right\| \text {, }
$$

where

$$
\phi_{i k}=\left\{\begin{array}{l}
\phi_{22}=\phi_{33}=\frac{1-C^{2}}{1+C^{2}} \\
\phi_{14}=\phi_{41}= \pm \frac{2 C}{1+C^{2}}
\end{array}\right.
$$


Here $C=\frac{g_{\otimes}-g_{\oplus}}{g_{\otimes}+g_{\oplus}}, g_{\otimes}, \quad g_{\oplus}$ - the indices of absorption of left- $(\otimes)$ and right- $(\oplus)$ circularly polarized components of the light beam amplitude.

\subsection{Mechanisms of phase anisotropy}

Amino acids and polypeptide circuits (initial structure of the protein) formed by them possess optical activity and are characterized by the following matrix operator

$$
\{\Omega\}=\left\|\begin{array}{cccc}
1 & 0 & 0 & 0 \\
0 & \omega_{22} & \omega_{23} & 0 \\
0 & \omega_{32} & \omega_{33} & 0 \\
0 & 0 & 0 & 1
\end{array}\right\|,
$$

where

$$
\omega_{i k}=\left\{\begin{array}{l}
\omega_{22}=\omega_{33}=\cos 2 \theta ; \\
\omega_{23}=-\omega_{32}=\sin 2 \theta .
\end{array}\right.
$$

Here $\theta$ - the rotation angle of polarization plane transformed by the protein circuit of light beam.

Fibrillar (secondary structure) protein networks formed by polypeptide circuits possess linear birefringence and are quite completely described by the Mueller matrix:

$$
\{D\}=\left\|\begin{array}{cccc}
1 & 0 & 0 & 0 \\
0 & d_{22} & d_{23} & d_{24} \\
0 & d_{32} & d_{33} & d_{34} \\
0 & d_{42} & d_{43} & d_{44}
\end{array}\right\|,
$$

where

$$
d_{i k}=\left\{\begin{array}{l}
d_{22}=\cos ^{2} 2 \rho+\sin ^{2} 2 \rho \cos \delta \\
d_{23}=d_{32}=\cos 2 \rho \sin 2 \rho(1-\cos \delta) \\
d_{33}=\sin ^{2} 2 \rho+\cos ^{2} 2 \rho \cos \delta \\
d_{24}=-d_{42}=\sin 2 \rho \sin \delta \\
d_{34}=-d_{43}=\cos 2 \rho \sin \delta \\
d_{44}=\cos \delta
\end{array}\right.
$$

Here $\rho$ - direction of fibril packing, $\delta$ - phase shift between linearly polarized orthogonal components of the light beam amplitude.

It is well known that the mechanisms of optically anisotropic absorption (relations (1)-(4)) that determine the laser fluorescence, sufficiently depend on the optical radiation wave length and are extremely manifested in the ultraviolet domain of the spectrum ${ }^{22-24}$. In the visible domain the mechanism of linear dichroism (relations (3), (4)) with the following fibrillar linear birefringence (relations (7), (8)) appears to be principal. In other words,

$$
\delta, \Delta \tau>>\theta, C \text {. }
$$


It follows from condition (9) that the matrix operator characterizing the mechanisms of linear dichroism and birefringence of fibrillar protein networks can be written as:

$$
\{M\}=\{D\}\{\Psi\}=\left\|\begin{array}{cccc}
1 & M_{12} & M_{13} & M_{14} \\
M_{21} & M_{22} & M_{23} & M_{24} \\
M_{31} & M_{32} & M_{33} & M_{34} \\
M_{41} & M_{42} & M_{43} & M_{44}
\end{array}\right\| .
$$

The process of absorption of laser radiation and the further phase modulation of fluorescence of protein molecules oriented along the direction of fibrils packing can be described by the vector-parametric equation

$$
S^{*}=\{M\} S\left(\alpha_{0}\right)
$$

In the developed form the Stokes vector of the beam $S^{*}$ transformed by the object is written as

$$
S^{*}=\left(1+M_{12}+M_{13}\right)^{-1}\left(\begin{array}{c}
1 \\
M_{21}+M_{22} \cos 2 \alpha_{0}+M_{23} \sin 2 \alpha_{0} \\
M_{31}+M_{32} \cos 2 \alpha_{0}+M_{33} \sin 2 \alpha_{0} \\
M_{41}+M_{42} \cos 2 \alpha_{0}+M_{3} \sin 2 \alpha_{0}
\end{array}\right),
$$

where

$$
\begin{aligned}
& \alpha^{*}=0,5 \operatorname{arctg}\left(\frac{M_{31}+M_{32} \cos 2 \alpha_{0}+M_{33} \sin 2 \alpha_{0}}{M_{21}+M_{22} \cos 2 \alpha_{0}+M_{23} \sin 2 \alpha_{0}}\right) \\
& \beta^{*}=0,5 \arcsin \left(\frac{M_{41}+M_{42} \cos 2 \alpha_{0}+M_{43} \sin 2 \alpha_{0}}{1+M_{12}+M_{13}}\right) .
\end{aligned}
$$

Here $\alpha^{*}$ - azimuth; $\beta^{*}$ - polarization ellipticity of the beam transformed by the object

It follows from the analysis of (12) - (14) that by varying the polarization azimuth $\alpha_{0}$ of the probing beam it is possible to minimize the impact of linear dichroism. It is easy to notice that at the fulfillment of the condition

$$
\alpha_{0}^{*}=\rho \pm 0.25 \pi \rightarrow \Delta \tau=1
$$

the matrix $\{\Psi\}$ (relation (1)) is transformed into a diagonal $\left\{\varphi_{i i}=1\right\}$.

Minimization of polarization ellipticity becomes the indicator of condition (15) realization

$$
\beta_{\min }^{*}=0,5 \arcsin \left(d_{42} \cos 2 \alpha_{0}^{*}+d_{43} \sin 2 \alpha_{0}^{*}\right) .
$$

On the other hand, physical condition (15) can be realized in the red $(\lambda=0.63 \mu \mathrm{m})$ part of the spectrum.

Thus, after measuring the elements of matrix operator $\{D(\lambda=0.63 \mu \mathrm{m})\}$, it is possible to determine the polarizationally optimal condition of the laser beam probing the biological layer in the blue part of the spectrum.

As a result of the performed model analysis there arise topical tasks:

- to perform the analysis of effectiveness of the conventional method of polarization mapping in the "red" $(\lambda=0.63 \mu \mathrm{m})$ range of the spectrum;

- to determine the diagnostic effectiveness of the method of laser polarization fluorescence $I\left(\alpha_{0}^{*}\right)$ on the base of (15). 


\section{OBJECTS, METHODS OF INVESTIGATION AND ALGORITHMS OF PROCESSING OF POLARIZATION MAPS OF LASER AND MUELLER-MATRIX IMAGES OF HISTOLOGICAL SECTIONS}

\subsection{Objects of Investigation}

Optically thin (attenuation coefficient $\tau<0.1$ ) histological sections of uterus neck of the following two types were used as objects of investigation:

- pre-cancer (dysplasia) condition - group 1 (19 samples);

- malignant tumor (adenocarcinoma) - group 2 (18 samples).

Histological sections were prepared according to standard technique on the freezing microtome. For each of such layers the presence of optically anisotropic protein networks - fibrillar collagen and muscle fibers - is typical. Optical properties of collagen fibrils are similar to those of a uniaxial crystal with optical axis directed along the axis of the fiber. According to the estimates made $\mathrm{in}^{25}$ the difference between the main refraction indices $\Delta \mathrm{n}$ is about $2.5 \times 10^{-3}$. Birefringence and dichroism of the network of muscle fibers are also determined by their fibrillar structure. The parts of muscle fibers consisting of unidirectionally oriented molecules of myosin manifest optical anisotropy. $\mathrm{In}^{26}$ the following estimate of birefringence of muscle tissue is presented: $\Delta \mathrm{n}=1.4 \times 10^{-3}$.

\subsection{The Technique of Measuring and Algorithms of Experimental Data Processing}

The coordinate distributions of polarization ellipticity in the plane of laser images of histological sections of uterus neck wall biopsy were measured in the setup of the standard Stokes-polarimeter. The detailed description of the optical scheme and main units of the experimental setup is presented in the series of research works ${ }^{11-14}$.

The values of polarization ellipticity within each pixel of CCD-camera were determined by the following algorithm

$$
\beta=0.5 \arcsin \frac{S_{4}}{S_{1}}=0.5 \arcsin \frac{I_{\otimes}-I_{\oplus}}{I_{\otimes}+I_{\oplus}} .
$$

Here $S_{i=1 ; 4}$ - parameters of the Stokes-vector in the points of digital image of a histological section: $I_{\otimes}, I_{\oplus}$ - intensities of left- and right-circularly polarized components of laser radiation.

In this research we used the "blue" laser with the wave-length $\lambda=0,412 \mu m$ and power $W=50 \mu W$ for exciting fluorescence. To perform the spectral division of polarization fluorescence, the interference filter with the transparency maximum $\lambda_{\max }=\mu m$ was located in front of CCD-camera, which was coordinated with maximal achieved intensity of laser polarization fluorescence in the given conditions.

Plane-polarized laser beams with polarization azimuths $\alpha_{0}=0^{0} ; \alpha_{0}^{*} ; 90^{0}$ were used as probing radiation exciting laser fluorescence of protein molecules. For each of these beams the coordinate distribution of laser fluorescence intensity $I(m \times n)$ was registered.

The objective estimation of the coordinate distributions $q \equiv\left\{\begin{array}{l}\beta(m \times n) ; \\ I(m \times n)\end{array}\right.$ was performed by means of the methods of statistical $^{11,12}$, correlation ${ }^{15-17}$ and fractal ${ }^{18,19}$ analysis.

\subsubsection{Statistical Analysis}

The ensemble of statistical moments of the $1^{\text {st }}-4^{\text {th }}$ order characterizing the distributions $q$ was calculated using the algorithms

$$
Z_{1}=\frac{1}{N} \sum_{j=1}^{N}|q| ; Z_{j}=\sqrt{\frac{1}{N} \sum_{j=1}^{N}(q)_{j}{ }^{2}} ; Z_{3}=\frac{1}{Z_{2}{ }^{3}} \frac{1}{N} \sum_{j=1}^{N}(q)_{j}^{3} ; Z_{4}=\frac{1}{Z_{2}{ }^{4}} \frac{1}{N} \sum_{j=1}^{N}(q)_{j}{ }^{4} .
$$

$N$ - the number of pixels of CCD-camera. 


\subsubsection{Correlation Analysis}

The structure of coordinate distributions $q(m \times n)$ was analyzed by means of autocorrelation method. Within each line of the array $(m \times n)$ of pixels the series of autocorrelation functions was determined

$$
\left\{G_{X X}=\left(\begin{array}{c}
G_{q=1, \ldots, n}^{(1)}(\Delta n) \\
G_{q=1, \ldots, n}^{(2)}(\Delta n) \\
\cdot \\
G_{q=1, \ldots, n}^{(m)}(\Delta n)
\end{array}\right) .\right.
$$

From (19) the averaged by the number of pixels array autocorrelation distribution was calculated

$$
\bar{G}_{X X}=\frac{\sum_{j=1}^{m} G_{X X}^{(j)}}{m} .
$$

Distributions (20) were characterized by the statistical moment of the $4^{\text {th }}$ order (relations (18)) that determined the "sharpness" of the peak or the speed of decrease of autocorrelation dependencies $\bar{G}_{X X}$. Further the above mentioned statistical parameter will be referred to as correlation moment $K$.

\subsubsection{Fractal Analysis}

Fractal analysis of the distributions $q(m \times n)$ was based on the calculation of logarithmic dependencies $\log J(q)-\log d^{-1}$ of power spectra $J(q)$ where $v=d^{-1}$ are spatial frequencies determined by geometrical sized $(d)$ of the structural elements of Mueller-matrix rotation invariants.

The dependencies $\log J(q)-\log d^{-1}$ were approximated by the least squares technique into the curves $V(\eta)$ and classified according to the following criteria ${ }^{23}$ :

- $q(m \times n)$ - fractal or self-similar if there is a constant inclination angle $\eta=$ const within 2 - 3 decades of the sizes $d$ change;

- $q(m \times n)$ - multifractal if there are several inclination angles $V(\eta)$;

- $q(m \times n)$ - random if there are no stable inclination angles $V(\eta)$ on the whole interval of the sizes $d$ change.

All the distributions $\log J(q)-\log d^{-1}$ were characterized by the statistical moment of the $2^{\text {nd }}$ order (relations (17)). This parameter will be further referred to as spectral moment $\Omega$.

\section{ANALYSIS AND DISCUSSION OF THE EXPERIMENTAL DATA}

Fig. 1 presents the series of experimentally measured coordinate distributions of polarization ellipticity in the points of laser $(\lambda=0,63 \mu \mathrm{m})$ images of histological sections of biopsy of uterus neck tissue of group 1 (fragments (1)-(4)) and group 2 (fragments (5)-(8)).

The comparative analysis of the data obtained reveals generally individual and at the same time - rather similar statistical (fragments (3),(7)), correlation (fragments (4),(9)) and scale-self-similar (fragments (5),(10)) structure of the coordinate distributions of polarization ellipticity (fragments $(1),(2)$ и (6),(7)) of laser images of the samples of both groups. This statement illustrates the similarity of histograms $N(\beta)$ of distributions $\beta$ with narrowly localized main extreme (fragments $(3),(7))$. 


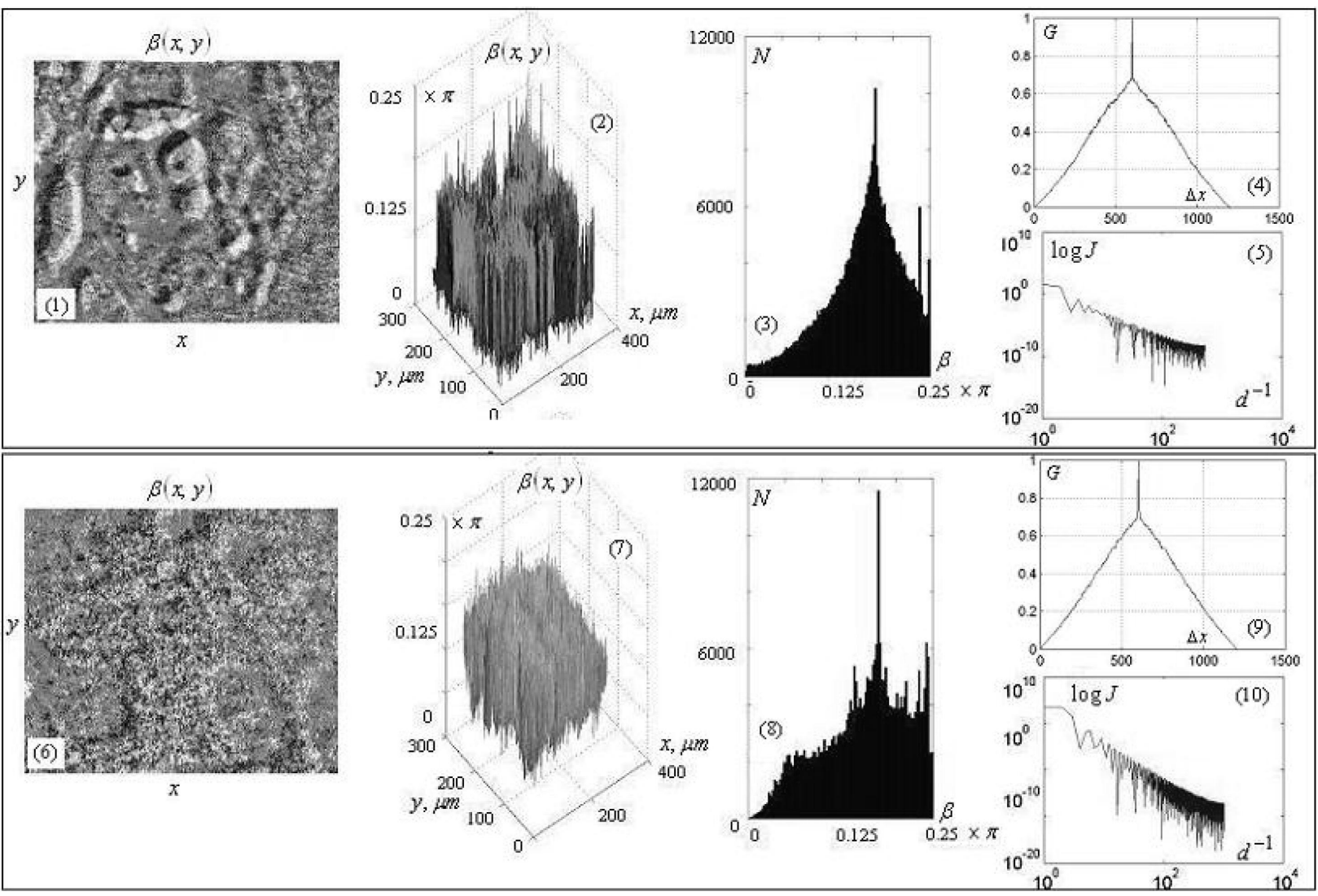

Figure 1. Polarization maps of laser images of histological sections of uterus neck tissue sections of group 1 (fragments (1)-(5)) and group 2 (fragments (6)-(10)). See explanations in the text.

The availability and closeness of localization of such extremes can be related to the similarity of optical geometrical structure (relations (7)) of fibrillar protein networks of the samples of both types. This fact is manifested in practically identical dependencies of autocorrelation functions (fragments (4),(9)) characterizing the peculiarities of coordinate phase modulation $\delta_{\min } \leftrightarrow \delta_{\max }$ (relations (8)) in the plane of histological sections of uterus neck tissue biopsy. Besides, the coordinate distributions $\beta$ are practically fractal - for $\log J(\beta)-\log d^{-1}$ dependencies the stable inclination angle of the approximating curves $V(\eta)$ on the whole range of changes of geometrical sizes is typical (fragments (5),(10)).

The difference between polarization maps of the samples of group 1 and group 2 are quantitatively illustrated in Table 1 .

It can be seen from the data obtained that the statistical moments of the $3^{\text {rd }}$ and $4^{\text {th }}$ order proved to be the most sensitive in the task of differentiation of polarization maps of the samples of both groups of samples. The difference between the above mentioned statistical parameters lies within $1,5\left(Z_{4}\right)$ to $2\left(Z_{3}\right)$ times - in grey color in Table 1.

In both groups of histological sections during the statistical, correlation and fractal approaches sensitivity ( $R=\frac{a}{a+b} 100 \%$ ) and specificity ( $S=\frac{c}{c+d} 100 \%$ ) of the polarization mapping method were determined, where $a$ and $b$ - the number of the correct and incorrect diagnoses within group $1 ; c$ and $d$ - the same for group 2 - Table 2. 
Table 1. Statistical $\left(Z_{i=1 ; 2 ; 3 ; 4}\right)$, correlation $(K)$ and fractal $(\Omega, V(\eta))$ parameters of distributions of polarization ellipticity in laser images of histological sections of uterus neck of group 1 (dysplasia) and group 2 (adenocarcinoma).

\begin{tabular}{|c|c|c|}
\hline \multirow{2}{*}{ Parameters } & \multicolumn{2}{|c|}{$\beta(m \times n)$} \\
\cline { 2 - 3 } & Dysplasia & Cancer \\
\hline$Z_{1}$ & $0.51 \pm 0.062$ & $0.59 \pm 0.071$ \\
\hline$Z_{2}$ & $0.21 \pm 0.037$ & $0.25 \pm 0.019$ \\
\hline$Z_{3}$ & $0.87 \pm 0.14$ & $1.68 \pm 0.25$ \\
\hline$Z_{4}$ & $1.41 \pm 0.19$ & $2.18 \pm 0.33$ \\
\hline$K$ & $1.68 \pm 0.24$ & $1.84 \pm 0.28$ \\
\hline$\Omega$ & $2.17 \pm 0.29$ & $2.46 \pm 0.34$ \\
\hline$V(\eta)$ & fractal & fractal \\
\hline
\end{tabular}

Table 2. Sensitivity and Specificity of the Method of Polarization Mapping of Uterus Neck Samples.

\begin{tabular}{|c|c|c|c|}
\hline Parameters & $Z_{i=1 ; 2 ; 3 ; 4}$ & $K$ & $\Omega$ \\
\hline$R, \%$ & 60 & 50 & 50 \\
\hline$S, \%$ & 52 & 50 & 50 \\
\hline
\end{tabular}

Thus, the statistical, correlation and fractal analyses of distributions of polarization ellipticity conditioned by the mechanisms of linear birefringence appeared to by not effective enough in the task of differential diagnostics of pre-cancer and malignant changes of uterus neck.

The potentiality of the method of polarization laser fluorescence is illustrated by the data presented in Figures 2 and 3. Here the coordinate distributions of fluorescence intensity $I$ (fragments (1),(5),(9)), histograms $N(I)$ (fragments (2),(6),(10)), autocorrelation functions $G(\Delta x)$ (fragments (3),(7),(11)) and logarithmic dependencies $\log J(q)-\log d^{-1}$ (fragments (4),(8),(12)) of such distributions are presented, which were obtained for different polarization states of the probing beam of the blue laser $(\lambda=0.41 \mu \mathrm{m})$.

The analysis of the data presented in Fig. 2 and Fig. 3 revealed:

1. The sufficient dependence of laser fluorescence of protein molecules of fibrillar networks on the polarization state of the laser beam probing the histological sections that reaches its extreme level (fragments (9)) for optimal polarization state $\alpha_{0}^{*}$ calculated using relation (15).

2. The shift of the main extremes of histograms $N(I)$ in the domain of greater values of fluorescence intensity for $\alpha_{0} \rightarrow \alpha_{0}^{*}$ (Fig. 2, fragments (2),(6),(10)).

3. Practically identical correlation (Fig. 2, fragments (3),(7),(11)) and multifractal (Fig. 2, fragments (4),(8),(12)) structure of coordinate distributions $I\left(\alpha_{0}\right)$ of the samples of uterus neck in the state of dysplasia.

4. Increase of coordinate homogeneity (smoother decrease of autocorrelation dependencies - Fig. 2, fragments (3),(7),(11)) and transformation of multifractal distributions $I\left(\alpha_{0}\right)$ into the fractal ones (formation of the stable inclination angle of the approximating curve - Fig. 2, fragments (4),(8),(12)) of the samples of uterus neck of group 2 for $\alpha_{0} \rightarrow \alpha_{0}^{*}$. 


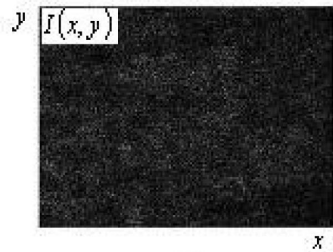

(1)

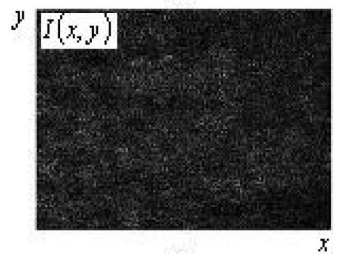

(5)

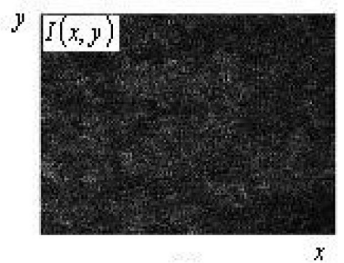

(9)

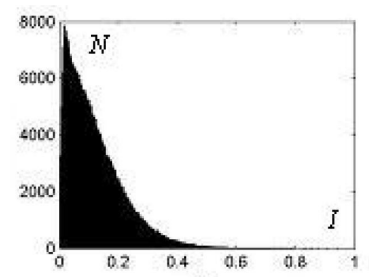

(2)

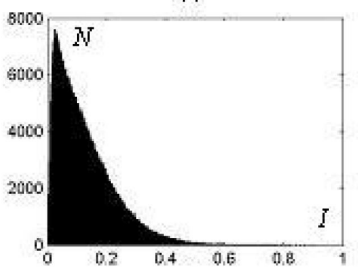

(6)

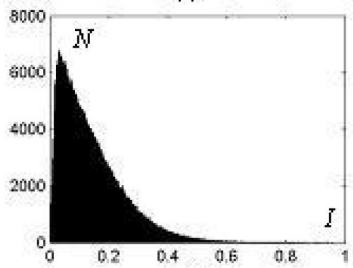

(10)

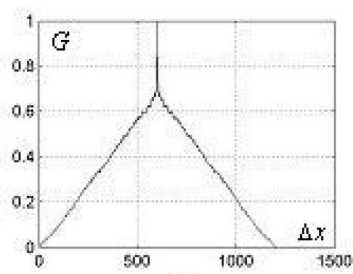

(3)

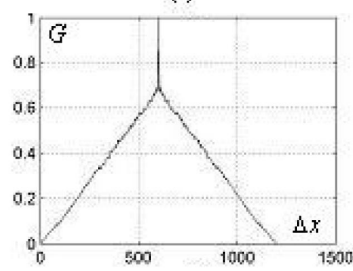

(7)

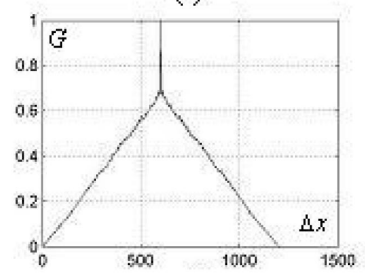

(11)

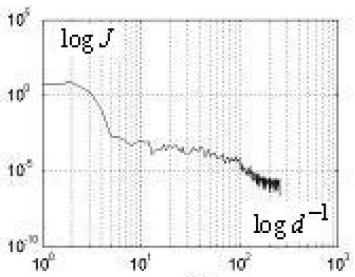

(4)

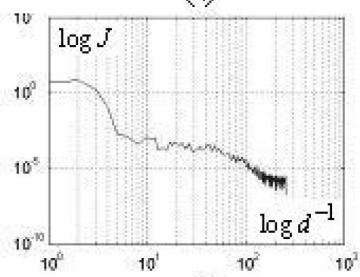

(8)

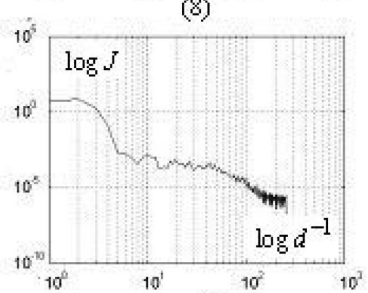

(12)

Figure 2. Coordinate, statistical, correlation and fractal distributions of laser fluorescence intensity of the samples of uterus neck tissue in pre-cancer state for the following polarization states: $\alpha_{0}=0^{0}$ - fragments (1)-(4); $\alpha_{0}=90^{0}$ - fragments (5)-(8); $\alpha_{0}=\alpha_{0}^{*}$ - fragments (9)-(12).

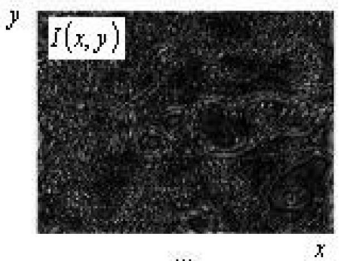

(1)

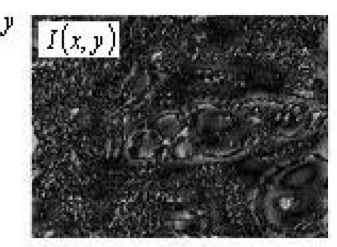

(5)

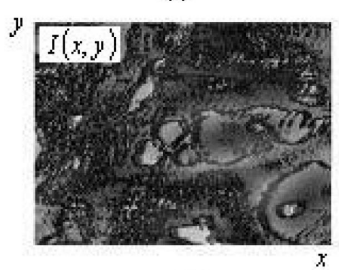

(9)

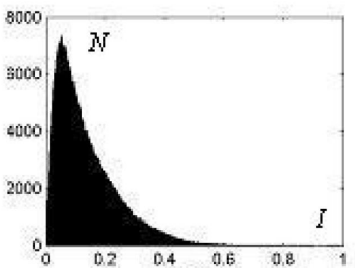

(2)

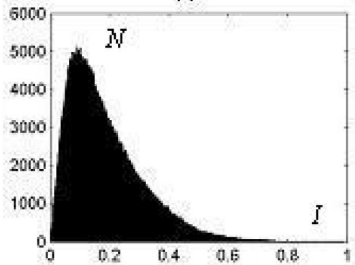

(6)

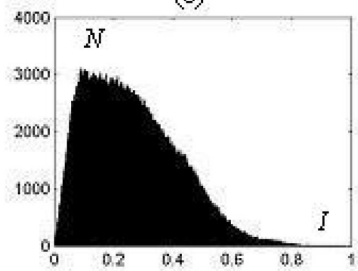

(10)

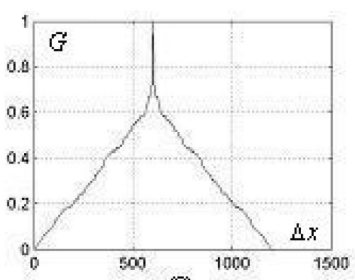

(3)

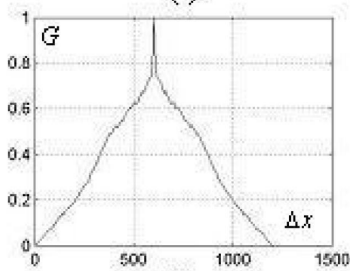

(T)

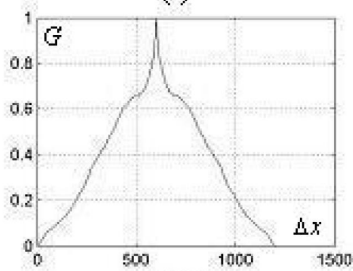

(11)
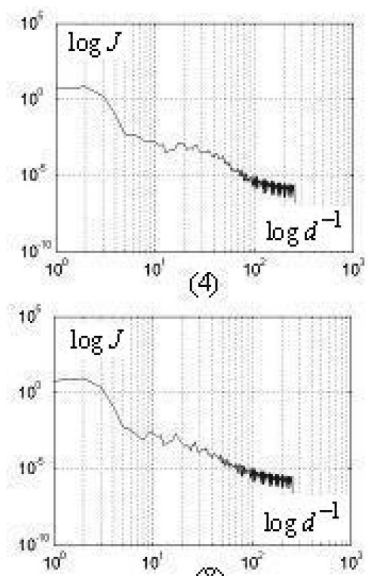

(8)

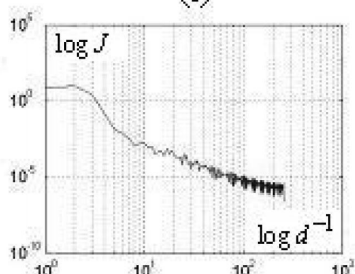

$(12)$

Figure 3. Coordinate, statistical, correlation and fractal distributions of laser fluorescence intensity of the samples of uterus neck tissue with adenocarcinoma for the following polarization states: $\alpha_{0}=0^{0}$ - fragments (1)-(4); $\alpha_{0}=90^{0}$ - fragments (5)-(8); $\alpha_{0}=\alpha_{0}^{*}$ - fragments (9)-(12). See explanations in the text. 
The difference between the coordinate distribution of laser polarization intensity of fluorescence $I\left(\alpha_{0}^{*}\right)$ of the protein networks of the samples of groups 1 and 2 is quantitatively illustrated by Table 3 .

Table 3. Statistical ( $Z_{i=1 ; 2 ; 3 ; 4}$ ), correlation $(K)$ and fractal $\left(\Omega, V(\eta)\right.$ ) parameters of distributions $I\left(\alpha_{0}^{*}\right)$ of histological sections of uterus neck of group 1 (dysplasia) and group 2 (adenocarcinoma).

\begin{tabular}{|c|c|c|}
\hline \multirow{2}{*}{ Parameters } & \multicolumn{2}{|c|}{$I\left(\alpha_{0}^{*}\right)$} \\
\cline { 2 - 3 } & Dysplasia & Cancer \\
\hline$Z_{1}$ & $0.12 \pm 0.016$ & $0.22 \pm 0.071$ \\
\hline$Z_{2}$ & $0.09 \pm 0.013$ & $0.16 \pm 0.029$ \\
\hline$Z_{3}$ & $0.38 \pm 0.054$ & $1.16 \pm 0.18$ \\
\hline$Z_{4}$ & $1.14 \pm 0.17$ & $0.54 \pm 0.11$ \\
\hline$K$ & $2.26 \pm 0.29$ & $0.78 \pm 0.13$ \\
\hline$\Omega$ & $1.47 \pm 0.21$ & $2.24 \pm 0.37$ \\
\hline$V(\eta)$ & multifractal & fractal \\
\hline
\end{tabular}

The similar comparative investigations were performed for other (polarizationally non-optimal) polarization states $\left(\alpha_{0}=0^{0} ; 90^{0}\right.$ ) of the probing laser beam. Exciting laser interference by the beam with polarization state $\alpha_{0}^{*}$ optimized using relation (15), appeared to be the most diagnostically sensitive. During the process of research, the following quantitative criteria of differentiation of benign and malignant changes were determined:

- difference between the values of the set of statistical moments of the $1^{\text {st }}-4^{\text {th }}$ order characterizing the distributions $I\left(\alpha_{0}^{*}\right)$, make up 1.9 times for $Z_{1}, 1.8$ times for $Z_{2}, 3$ times for $Z_{3}$ and 2 times for $Z_{4}$;

- for oncological state of uterus neck tissue the correlation moment decreases by more than 3 times;

- the coordinate structure of distributions $I\left(\alpha_{0}^{*}\right)$ of the samples of group 2 is fractal, while the spectral moment $\Omega$ characterizing the logarithmic dependencies $\log I\left(\alpha_{0}\right)-\log d^{-1}$ increases by 1.5 times.

During the statistical, correlation and fractal approaches the sensitivity and specificity of the method of laser polarization fluorescence were determined within both groups of histological sections - Table 4 .

Table 4. Sensitivity and Specificity of the Method of Polarization Mapping of Uterus Neck Samples.

\begin{tabular}{|c|c|c|}
\hline Parameters & $R, \%$ & $S, \%$ \\
\hline$Z_{i=1 ; 2 ; 3 ; 4}$ & 60 & 52 \\
\hline$K$ & 50 & 50 \\
\hline$\Omega$ & 50 & 50 \\
\hline
\end{tabular}

Thus, the statistical, correlation and fractal analysis of Mueller-matrix invariants of laser polarization fluorescence conditioned by the mechanisms of linear and circular dichroism proved to be effective for the task of differential diagnostics of benign and malignant changes of uterus wall. 


\section{CONCLUSION}

1. The model of laser polarization fluorescence of biological tissues considering the mechanisms of optically anisotropic absorption - linear and circular dichroism of protein networks was suggested.

2. Mueller-matrix rotation invariants characterizing polarization manifestations of laser fluorescence are determined.

3. The interconnections between the statistical, correlation and fractal parameters characterizing the Mueller-matrix images of laser polarization fluorescence and the peculiarities of the mechanisms of optically anisotropic absorption of histological sections of uterus wall biopsy were found.

4. Effectiveness of the method of azimuth-invariant Mueller-matrix mapping of laser polarization fluorescence of protein networks in the task of differentiation of benign and malignant tumors of uterus wall was demonstrated.

\section{REFERENCES}

[1] Bueno, J.M. and Jaronski, J., "Spatially resolved polarization properties for in vitro corneas," Ophthal., Physiol., Opt., 21, 384-392 (2001).

[2] Bueno, J.M. and Vargas-Martin, F., "Measurements of the corneal birefringence with a liquid-crystal imaging polariscope," Applied Optics 41, 116-124 (2002).

[3] Bueno, J.M. and Campbell, M.C.W., "Polarization properties of the in vitro old human crystalline lens," Ophthal., Physiol., Opt., 23, 109-118 (2003).

[4] Tower, T.T. and Tranquillo, R.T., "Alignment Maps of Tissues: I., Microscopic Elliptical Polarimetry," Biophys., J., 81, 2954-2963 (2001).

[5] Tower, T.T. and Tranquillo, R.T., "Alignment Maps of Tissues: II., Fast Harmonic Analysis for Imaging," Biophys., J., 81, 2964-2971 (2001).

[6] Shribak, M., Oldenbourg, R., "Techniques for fast and sensitive measurements of two-dimensional birefringence distributions," Appl. Opt., 42, 3009-3017 (2003).

[7] Smith, M.H., Burke, P., Lompado, A., Tanner, E. and Hillman, L.W., "Mueller matrix imaging polarimetry in dermatology," Proc. SPIE 3991, 210-216 (2000).

[8] Smith, M.H., "Interpreting Mueller matrix images of tissues," Proc., SPIE 4257, 82-89 (2001).

[9] Wang, X. and Wang, L.V., "Propagation of polarized light in birefringent turbid media: A Monte Carlo study," J. Biomed. Opt., 7, 279-290 (2002).

[10] Lu, S. and Chipman, R. A., "Interpretation of Mueller matrices based on polar decomposition," J. Opt. Soc. Am., A 13, 1106-1113 (1996).

[11] Ushenko, A.G., Misevich, I.Z., Istratiy, V., Bachyns'ka, I., Peresunko, A.P., Numan, O.K. and Moiysuk, T.G., "Evolution of statistic moments of 2D-distributions of biological liquid crystal net Mueller matrix elements in the process of their birefringent structure changes," Advances in Optical Technologies, 423145 (2010).

[12]Ushenko, Yu. A., Peresunko, A.P. and Bozan Adel Baku, "A New Method of Mueller-Matrix Diagnostics and Differentiation of Early Oncological Changes of the Skin Derma," Advances in Optical Technologies, 952423 (2010).

[13] Angelsky, O.V. and Ushenko, Yu.A., "The Degree of Mutual Anisotropy of Biological Liquid Crystals Net during the Diagnostics of Human Tissues Birefringence," Advances in Optical Technologies, 321275 (2010).

[14] Angelsky, O.V., Ushenko, Yu.A., Dubolazov, A.V. and Telenha, O.Yu., "The Interconnection between the Coordinate Distribution of Mueller-Matrixes Images Characteristic Values of Biological Liquid Crystals Net and the Pathological Changes of Human Tissues," Advances in Optical Technologies, 130659 (2010).

[15] Ushenko, Yu.A., Tomka, Yu.Ya. and Dubolazov, A.V., "Laser diagnostics of anisotropy in birefringent networks of biological tissues in different physiological conditions", Quantum Electron 41, 170-175 (2011).

[16] Ushenko, Yu.O., Tomka, Yu.Ya., Misevitch, I.Z., Istratiy, V.V. and Telenga, O.I., "Complex degree of mutual anisotropy of biological liquid crystals nets", Opt. Eng., 50, 039001 (2011).

[17] Ushenko, Yu.A., Tomka Yu.Ya. and Dubolazov, A.V., "Complex degree of mutual anisotropy of extracellular matrix of biological tissues," Optics and Spectroscopy 110, 814-819 (2011).

[18] Ushenko, Yu.A., Tomka, Yu.Ya., Dubolazov, A.V., Telenga, O.Yu., "Diagnostics of optical anisotropy changes in biological tissues using Müller matrix", Quantum Electron, 41, 273-277 (2011). 
[19] Ushenko, Yu.A., Telenga, O.I., Peresunko A.P. and Numan, O.K., "New parameter for describing and analyzing the optical-anisotropic properties of biological tissues," J., Innov., Opt., Health Sci., 4, 463-475 (2011).

[20] Ushenko, Yu.A., "Investigation of formation and interrelations of polarization singular structure and Muellermatrix images of biological tissues and diagnostics of their cancer changes," J., Biomed., Opt., 16, 066006 (2011).

[21] Ushenko, A.G., Burkovets, D.N. and Ushenko, Yu.A., "Polarization-Phase Mapping and Reconstruction of Biological Tissue Architectonics during Diagnosis of Pathological Lesions," Optics and Spectroscopy 93, 449456 (2002).

[22] Angel'skii, O.V., Ushenko, A.G., Arkhelyuk, A.D., Ermolenko, S.B., Burkovets, D.N. and Ushenko, Yu.A., "Laser Polarimetry of Pathological Changes in Biotissues," Optics and Spectroscopy 89, 973-978 (2000).

[23] Yermolenko, S., Ushenko, A., Ivashko, P., Goudail, F., Gruia, I., Zimnyakov, D. and Mikhailova, A., "Spectropolarimetry of cancer change of biotissues," Proc. SPIE 7388, 73881D (2009).

[24] Angelsky, O.V., Tomka, Yu.Ya., Ushenko, A.G., Ushenko, Ye.G., Yermolenko, S.B. and Ushenko, Yu.A., "2D tomography of biotissues images in pre-clinic diagnostics of their pre-cancer states," Proc. SPIE 5972, $59720 \mathrm{~N}(2005)$

[25] Gathercole, L.J. and Keller A., "Light microscopic waveforms in collagenous tissues and their structural implications," Colston Papers 26, 153-187 (1975).

[26] de Boer, J. F. and Milner, T.E., "Review of polarization sensitive optical coherence tomography and Stokes vector determination,” J. Biomed. Opt., 7, 359-371 (2002). 